\title{
Interdependência dos preços do milho no sul brasileiro
}

\author{
Eliane Pinheiro de Sousa ${ }^{1}$ \\ Marcelo José Braga ${ }^{2}$ \\ Dênis Antônio da Cunha ${ }^{3}$
}

Resumo: Este artigo objetiva verificar a relação entre os preços do milho nos estados do Paraná, do Rio Grande do Sul e de Santa Catarina, buscando testar se a Lei do Preço Único prevaleceu nesses mercados, no período de outubro de 2002 a março de 2009. Utilizaram-se dados mensais provenientes do Centro de Estudos Avançados em Economia Aplicada (CEPEA), da ESALQ / USP. Os métodos empregados compreendem os testes de raiz unitária e de cointegração de Johansen, estimação da função impulso-resposta, decomposição da variância dos erros de previsão, estimação do modelo vetorial de correção de erro (VEC). Os resultados mostraram que a Lei do Preço Único não foi perfeitamente verificada nos mercados regionais de milho analisados, quando foram impostas restrições ao coeficiente de relacionamento de longo prazo.

Palavras-chave: relação entre preços; Lei do Preço Único; mercado regional de milho.

\section{Interdependece of maize prices in Southern Brazil}

\begin{abstract}
This article aims to verify the relationship between the prices of the maize in the states of Paraná, Rio Grande do Sul and Santa Catarina in order to test the validity of the Law of One Price at those markets over the October, 2002 to March, 2009 period. Monthly data were extracted from the Center of Advanced Studies for Applied Economy (CEPEA) of ESALQ / USP. The employed methods understand the tests for unitary root and Johansen's co-integration, impulse-response function, decomposition of the variance of the forecasting error and estimate of the vector

1 Doutoranda em Economia Aplicada da Universidade Federal de Viçosa (UFV) e Professora adjunta do Departamento de Economia da Universidade Regional do Cariri (URCA). E-mail: pinheiroeliane@hotmail.com 2 Ph.D em Economia e Professor associado do Programa de Pós-Graduação em Economia Aplicada da Universidade Federal de Viçosa (UFV). E-mail: mjbraga@ufv.br

3 Doutorando em Economia Aplicada da Universidade Federal de Viçosa (UFV)

E-mail: denisufv@gmail.com
\end{abstract}


error correction model (VEC). The results showed that the Law of One Price was not perfectly verified for the regional maize markets analyzed, when restrictions were imposed in the coefficient of relationship of long period.

Keywords: relation between prices; Law of One Price; regional maize market. JEL: C32, Q13

\section{Introdução}

A produção de milho no Brasil, juntamente com a da soja, contribui com cerca de $80 \%$ para a produção de grãos. Essas duas culturas se diferenciam pelo fato de a soja ter liquidez imediata, em virtude de suas características de commodity no mercado internacional, enquanto o milho tem sua produção destinada ao abastecimento interno. Entretanto, nos últimos vinte e oito anos, o milho tem evoluído como cultura comercial, registrando taxas de crescimento da produção de $3 \%$ ao ano e da área cultivada de 0,4\% ao ano (Duarte, 2007). Essa mudança na configuração do mercado brasileiro de milho e sua expansão no mercado externo podem ser atribuídas à crescente demanda dos Estados Unidos por milho para produção de etanol e à elevação das importações de milho pela China. Tais fatores têm refletido em aumentos significativos de seus preços nas bolsas internacionais (Perez et al. 2007). Nesse contexto, é relevante ressaltar que a agroenergia tem influenciado não apenas o mercado internacional de milho, mas outras commodities agrícolas que também podem ser utilizadas para produção de biocombustíveis como, por exemplo, a cana-de-açúcar, a mamona e a soja.

O milho é cultivado em todo o território nacional, e a região Sul, área de estudo deste trabalho, é a maior produtora do Brasil, perfazendo uma quantidade produzida de 24.020.568 toneladas, que corresponde a 46,09\% da produção nacional desse grão, conforme dados da Produção Agrícola Municipal, 2007 (IBGE 2007).

De acordo com Bittencourt e Barros (1996), avanços na produção somente implicarão em completa eficiência econômica se as principais regiões produtoras operarem de forma integrada, de modo que os eventuais déficits ou superávits reflitam os preços locais, sinalizando as desejadas movimentações de suprimentos, em curto prazo, e ajustes de produção, em médio e longo prazos. A importância da análise de integração também é ressaltada por Rosado (2006). Na concepção dessa autora, a integração facilita a tomada de decisão dos agentes envolvidos e contribui para aumentar a eficiência alocativa no mercado, além de possibilitar maior especialização e comércio, o que resulta em melhoria no bem-estar dos produtores e consumidores.

Nesse sentido, dada a relevância da integração dos mercados, é importante 
desenvolver estudos que busquem conhecer as interdependências dos preços de diferentes mercados de dada commodity. Segundo Silveira (2004), tais estudos contribuem para a formulação de políticas governamentais e para a tomada de decisão dos agentes acerca da produção e da comercialização.

Entretanto, o que se tem observado mais frequentemente na literatura econômica são estudos que contemplem a interdependência dos preços de commodities agrícolas entre mercados internos e externos. Com relação ao milho, isso é observado, por exemplo, nos estudos desenvolvidos por Costa e Ferreira Filho (2000), que analisaram outras commodities, além do milho, e Santos et al. (2007), que se preocuparam em verificar a relação de preços do milho entre os mercados doméstico e internacional. No entanto, a transmissão de preços também pode ser enfocada em uma análise regional, como é caso do trabalho de Alves et al. (2006), que abordaram essa temática para diversas commodities, dentre elas, o milho, no estado do Paraná, que é o maior produtor nacional. Além do estado do Paraná, Chiodi (2006) também considerou os outros dois estados da região Sul, assim como os estados de Mato Grosso, Mato Grosso do Sul, Goiás, São Paulo, Bahia e Minas Gerais, para analisar a dinâmica do processo de formação de preços no mercado regional de milho, entre 1996 a 2004.

O presente estudo segue a linha do trabalho de Chiodi (2006), mas considera apenas os estados sulinos. Além de realizar uma análise para um período mais recente, tendo em vista que esta pesquisa abrange o horizonte temporal de outubro de 2002 a março de 2009, acrescenta uma análise de decomposição da variância dos erros de previsão e função impulso-resposta, que não foram investigados por Chiodi (2006). Portanto, este trabalho pretende verificar se a Lei do Preço Único pode ser verificada entre esses estados da região Sul do país, ou seja, se variações de preços em um estado poderão ser repassadas aos demais.

O artigo está organizado em quatro seções, além desta introdução. Os referenciais teóricos e analíticos e a fonte dos dados compõem a segunda e terceira seções. Na próxima são apresentados e discutidos os resultados; e, na última, as conclusões.

\section{Formação de preços e políticas agrícolas nos mercados regionais de milho}

Para discutir o processo de formação de preços vigentes nos mercados regionais de milho, é relevante apresentar uma retrospectiva relativa à sua dinâmica e à adoção de instrumentos de políticas agrícolas. Conforme Chiodi (2006), a formação do preço do milho no mercado brasileiro até final dos anos 1980 se dava em uma economia fechada e com fortes intervenções governamentais. Dentre as políticas agrícolas intervencionistas, destaca-se a Política de Garan- 
tia de Preços Mínimos (PGPM), que objetivava garantir renda aos produtores e oferta adequada dos produtos. Tal política atuou em diversas regiões, sendo mais concentrada no Paraná.

A estrutura de formação de preços nacionais dos produtos agrícolas em geral e, do milho, em particular, modificou-se a partir dos anos 1990 com a abertura econômica. A partir de então, o movimento das cotações internacionais passa a influenciar o comportamento dos preços domésticos do milho. Nesse contexto e em face da menor atuação governamental no setor agrícola, surgem novas formas de apoio à agricultura, que propiciavam menores gastos ao setor público em relação à PGPM. Dentre esses instrumentos modernos de políticas agrícolas, ressaltam-se os Contratos de Opções de Venda e o Prêmio de Escoamento da Produção (PEP).

Conforme Conceição (2002), a aquisição de um Contrato de Opção de Venda por um produtor ou uma cooperativa requer um pagamento de um preço, o que assegura o direito de vender sua produção a um valor definido na data de vencimento do contrato. O PEP é empregado quando o preço de mercado encontra-se abaixo do preço mínimo, de forma que para garantir ao produtor o preço mínimo, o governo ao invés de comprar e estocar o excedente, paga aos agentes de comercialização um prêmio, cujo valor é determinado por meio de leilões e reflete a diferença entre o preço de mercado e o preço mínimo.

Esses instrumentos exerceram papel fundamental na comercialização do milho não apenas quando foram criados, mas no contexto atual. Conforme informações de mercado apresentados por Alves et al. (2009), aproximadamente 4,2 milhões de toneladas de milho foram beneficiadas por tais mecanismos. A influência dessas políticas é relevante nas regiões consideradas nesse estudo. Um exemplo disso é mostrado no Portal do Agronegócio (2009), em que tais políticas são requeridas pelo estado do Paraná para venda de seus estoques, visto que as constantes reduções de preço do milho prejudicaram sobremaneira a sua comercialização.

Outro aspecto importante que deve ser mencionado é a liderança do estado do Paraná como maior produtor nacional de milho. De acordo com dados da Produção Agrícola Municipal, apresentados pelo IBGE (2007), 27,36\% da produção brasileira de milho provêm desse estado. Os demais estados sulistas, Rio Grande do Sul e Santa Catarina, participam com 11,45\% e 7,28\%, respectivamente, da produção nacional. Entretanto, conforme mostrado por Chiodi (2006), esses dois últimos estados apresentam expressivo consumo de milho destinado principalmente para aves e suínos, fazendo com que sejam importadores do produto. Com base nessas evidências, considera-se que o estado do Paraná represente o mercado primário do milho, visto que se configura como principal praça de comercialização, e os estados do Rio Grande do Sul e de Santa Catarina atuem como mercados secundários. Para comprovar a influência do estado do Paraná na formação de preços do milho, ou seja, se os preços nesse estado estão integrados com os demais estados sulistas, 
faz-se necessário a realização de testes econométricos.

\section{Procedimentos metodológicos}

\subsection{Referencial teórico}

Este trabalho teve como fundamento teórico a Lei do Preço Único (LPU), que estabelece que em mercados concorrentes, isentos de custos de transporte e barreiras comerciais, bens idênticos devem ser vendidos por preços equivalentes, independente da moeda em que os preços são cotados (Krugman e Obstfeld 2005).

Segundo Ardeni (1989), a arbitragem de commodities assegura que cada bem possua um preço único, definida em uma unidade corrente comum. Nesse sentido, quando houver arbitragem, o preço tenderá a aumentar no mercado com menor preço, resultante do aumento da quantidade demandada, enquanto o preço tenderá a reduzir no mercado com preço mais elevado, em virtude do excesso da quantidade ofertada. Esse processo de arbitragem continuará até que os preços se igualem nos dois mercados.

Para Fackler e Goodwin (2001), a LPU pode ser manifestada nas seguintes formas: fraca, diante da condição de arbitragem espacial; agregada, enunciada em termos de índice de preço e conhecida como Paridade do Poder de Compra; e forte, considera que o comércio seja contínuo e assegura a condição de arbitragem. Essa última forma é a mais frequentemente testada.

A aplicabilidade da LPU tem sido defendida em diversos estudos entre diferentes países, como, por exemplo, os de Bungiorno e Uusivuori (1992) para produtos florestais entre Estados Unidos, Reino Unido, Alemanha, França, Itália, Bélgica e Japão, no período de 1978 a 1988; de Lima e Burnquist (1997) para os mercados de soja em grão no Brasil, Estados Unidos e Alemanha, no período de 1985 a 1995; e de Coelho (2004) para os mercados de algodão brasileiro e norte-americano na década de 1990. Esses estudos confirmaram a LPU por meio da utilização do instrumental metodológico de cointegração. Empregando esse mesmo procedimento econométrico, a proposição da LPU também é sustentada entre diferentes regiões de um mesmo país, como, por exemplo, os trabalhos empíricos de Jung e Doroodian (1994) para produtos florestais entre diferentes mercados norte-americanos, no período entre 1950 a 1985; de Nogueira et al. (2005), para mercados brasileiros de café arábica (Minas Gerais e São Paulo), no período de 1996 a 2000; e de Chiodi (2006), para mercados de milho (entre os estados do Centro-Oeste e entre os estados sulistas de Santa Catarina e do Rio Grande do Sul), no período entre 1996 a 2004 . 
Com base em Mundlak e Larson (1992), em termos matemáticos, a LPU pode ser expressa por:

$$
P_{i t}=\alpha+\beta P_{j t}+u_{t}
$$

em que $P_{i t}$ e $P_{j t}$ são preços logaritimizados de determinada commodity, nos mercados de duas regiões i e j, em dado período de tempo t; $\alpha$, uma constante (ou intercepto); e o coeficiente $\beta$ é a elasticidade de transmissão de preço entre essas regiões consideradas, de modo que variações de preço na região j serão plenamente transmitidas para a região $\mathrm{i}$, se o valor de $\beta$ for igual a um. Em contrapartida, variações de preço na região j não ocasionarão impactos na região $\mathrm{i}$, se o valor de $\beta$ for igual a zero.

\subsection{Referencial analítico}

Com o intuito de atender aos objetivos deste estudo, empregaram-se testes de raiz unitária, e de cointegração de Johansen; estimação da função de impulsoresposta; decomposição da variância dos erros de previsão; estimação e análise do modelo vetorial de correção de erro (VEC).

Quando as variáveis individuais não são estacionárias, mas uma combinação linear delas é estacionária, então diz-se que essas variáveis são cointegradas (Engle \& Granger 1987). Para testar a cointegração entre as séries de preços do milho no Sul brasileiro, deve-se primeiro determinar a ordem de integração, que pode ser obtida por meio do teste de raiz unitária. Dentre os testes convencionais de raiz unitária, o de Dickey-Fuller Aumentado (ADF) tem sido bastante utilizado na literatura. A hipótese nula deste teste refere-se à presença de uma raiz unitária. Essa hipótese deverá ser rejeitada, se o valor calculado da estatística t exceder seu valor crítico de Dickey-Fuller, indicando que a série é estacionária; caso contrário, a série é não estacionária.

Os testes tradicionais de raiz unitária desconsideram a presença de sazonalidade nas séries temporais, mas tais séries podem não ter um padrão uniforme de mudanças sazonais, resultando em uma rejeição errônea da hipótese de raiz unitária. Para evitar que isso aconteça e verificar se existe o componente de sazonalidade estocástica, deve-se proceder ao teste de raiz unitária sazonal, sendo que o mais frequentemente empregado é o teste HEGY (Hylleberg et al. 1990).

De acordo com Cunha (2008), quando o termo $\pi$, que representa um dos parâmetros da regressão a ser estimada para identificação da raiz unitária, for estatisticamente igual a zero, significa que a série possuirá uma raiz unitária regular e quando houver qualquer um dos outros $\pi \mathrm{i}$ 's $=\mathrm{O}(\mathrm{i}=2, \ldots, 12)$, sinalizará presença de raízes unitárias sazonais. Ademais, adota-se o teste t convencional para analisar a significância estatística de $\pi_{1}$ e $\pi_{2}$, enquanto utiliza-se o teste F para verificar a significância dos pares de raízes complexas, sendo que os 
valores críticos foram obtidos por Franses e Hobijn (1990).

Além disso, também é importante verificar se as séries analisadas registraram mudança estrutural durante o período enfocado, pois se houver alteração na estrutura comportamental e essa não for considerada, pode resultar em estimativas viesadas e com reduzido poder de previsão. Para tal, pode-se realizar o teste de Chow, que busca testar a hipótese nula de ausência de mudança estrutural, empregando um teste F especial, proposto por Gregory Chow em 1960. O emprego desse teste requer que seja definido o período da quebra, considerando as fases que antecedem e sucedem à mudança estrutural (Cunha, 2008). Os valores críticos foram obtidos por Lanne et al. (2002).

Após identificação da ordem de integração, se todas as séries tiverem a mesma ordem de integração, I(d) e existir um vetor $\beta(\neq 0)$, em que a combinação linear dessas variáveis seja de ordem $\mathrm{d}-\mathrm{b}, Z_{t}=\beta^{\prime} X_{t} \sim I(d-b), b>0$, então $\mathrm{Y}_{\mathrm{t}}$ será um vetor de cointegração de ordem $(\mathrm{d}, \mathrm{b})$, representada por $X_{t} \sim C I(d, b)$ conforme Engle e Granger (1987). Em outras palavras, a cointegração consiste em uma combinação linear de variáveis não estacionárias que possuem relação de convergência no longo prazo.

Existem diversos métodos para testar as relações de cointegração entre as variáveis, como, por exemplo, os de Engle e Granger, Stock e Watson e Johansen. Dentre esses três testes de cointegração, Gonzalo (1994) indicou o procedimento de Johansen como estatisticamente superior aos outros dois. Portanto, o método de Johansen é empregado neste trabalho para verificar todas as relações de longo prazo estabelecidas entre as séries de preços de milho nos estados do Sul brasileiro.

A estimação econométrica das relações de preços do milho entre os mercados regionais sulistas baseou-se no modelo de autorregressão vetorial (VAR), cuja representação do VAR, de ordem p, é dada por

$Y_{t}=\delta+\Theta_{1} Y_{t-1}+\ldots+\Theta_{p} Y_{t-p}+\varepsilon_{t}$,

em que cada $\Theta_{j}$ é uma matriz de parâmetros k x k, e $\varepsilon_{t}$ é um vetor k-dimensional de termos ruído branco com matriz de covariância $\Sigma$.

De acordo com Mayorga (2006), tais coeficientes não consideram as interdependências das variáveis contidas no modelo VAR. Dessa forma, os impactos das inovações podem ser avaliados por meio da função impulso-resposta, que delineia o comportamento das séries que fazem parte do modelo VAR em resposta a choques ocasionados por variáveis residuais.

Outra forma de caracterizar o relacionamento dinâmico entre as variáveis do modelo pode ser dada pela decomposição da variância dos erros de previsão para k períodos à frente. Segundo Sampaio et al. (2005), essa decomposição permite separar a variância dos erros de previsão acerca de cada variável do sistema em componentes que podem ser atribuídos à própria variável e às 
demais variáveis endógenas, ou seja , mostra, em termos percentuais, qual o efeito que um choque não antecipado sobre dada variável tem sobre ela própria e sobre as demais variáveis que fazem parte do sistema.

Conforme Bacchi (1994), se houver cointegração entre as variáveis, seu desvio relativo à trajetória de longo prazo deverá ser incluído no modelo através do mecanismo de correção de erro (VEC), que consiste em incorporar o termo de erro defasado de um período no modelo, com o intuito de recuperar as relações de longo prazo que foram perdidas na diferenciação das séries. Esse modelo pode ser descrito por

$\Delta Y_{t}=\delta+\Gamma_{1} \Delta Y_{t-1}+\ldots+\Gamma_{p-1} \Delta Y_{t-p+1}+\Pi Y_{t-1}+\varepsilon_{t}$,

em que $\Pi=\gamma \beta^{\prime}$, e as combinações lineares $\beta^{\prime} Y_{t-1}$ representam as r relações de cointegração.

Para Harris (1995), a principal vantagem de escrever o sistema em termos do modelo de correção de erro diz respeito à incorporação de informações tanto de curto quanto de longo prazo, via ajustes no $\Delta Y_{t}$, as quais são dadas pelas estimativas dos parâmetros $\Gamma_{i} \mathrm{e} \Pi$.

O número de vetores de cointegração depende do posto ou do rank (r) da matriz $\Pi$. De acordo com Verbeek (2000), podem-se ter as seguintes possibilidades:

(i) Se $r(\Pi)=0$, esta matriz será nula, logo, todos os componentes de $X_{t}$ serão I(1). Nessa situação, não haverá cointegração, já que não haverá combinação linear entre si que seja estacionária.

(ii) Se $r(\Pi)=k$, ou seja, o posto de $\Pi$ for completo, então as variáveis $X_{t}$ serão I(o). Isso significa que qualquer combinação linear entre as variáveis será estacionária em nível. Portanto, não se aplicará a cointegração.

(iii) Se o $<\mathrm{r}<\mathrm{n}$, ter-se-á a presença de $\mathrm{r}$ vetores de cointegração, que é a possibilidade que interessa a este trabalho.

Com o intuito de identificar o número de vetores de cointegração presentes neste artigo, empregaram-se os testes do traço e do máximo autovalor. Conforme Enders (1995), o primeiro visa testar a hipótese nula de que o número de vetores de cointegração distintos é menor ou igual a r, contra a hipótese alternativa de que o número desses vetores é maior do que r, enquanto o segundo busca testar a hipótese nula de que o número de vetores de cointegração é r, contra a hipótese alternativa de existência de r+1 vetores de cointegração. Em termos matemáticos, esses testes podem ser caracterizados por

$$
\lambda_{\text {trace }}(r)=-T \sum_{i=r+1}^{n} \ln \left(1-\lambda_{i}^{\prime}\right) \text {, }
$$

em que $\bar{\lambda}_{\mathrm{i}}$ são os valores estimados das raízes características obtidos da matriz 
П e T, número de observações.

$$
\lambda_{\text {max }}(r, r+1)=-T \ln \left(1-\lambda_{r+1}^{\prime}\right)
$$

Além disso, também se consideraram os testes de hipótese acerca dos parâmetros $\beta$. Segundo Coelho (2004), a existência de um vetor de cointegração não pode ser considerada condição suficiente para determinar a perfeita integração de mercado, nem para garantir que todas as séries participem do equilíbrio de longo prazo. Dessa forma, ele recomenda que sejam implementados testes de hipóteses sobre os parâmetros $\beta$.

De acordo com Johansen e Juselius (1990), citados por Coelho (2004), as hipóteses sobre os parâmetros $\beta$ apresentam o seguinte formato:

$$
-2 \log Q\left(H_{0}\right)=-T \sum_{i=1}^{r} \log \left[\frac{\left(1-\lambda_{i}^{*}\right.}{\left(1-\lambda_{i}\right.}\right], \text { para } \mathrm{i}=1, \ldots, \mathrm{r},
$$

em que $T$ é o número de observações; $r$, número de vetores de cointegração; e $\lambda_{i}^{*} \boldsymbol{e} \lambda_{i}$ representam os autovalores do modelo restrito e irrestrito, respectivamente.

As hipóteses nulas $\left(\mathrm{H}_{\mathrm{o}}\right)$, a serem testadas neste trabalho, são as seguintes:

$\beta_{\mathrm{PR}}=0, \quad \beta_{\mathrm{RS}}=0, \quad \beta_{\mathrm{PR}}=\beta_{\mathrm{RS}}$

sendo que as duas primeiras testam se os mercados de milho no Paraná e no Rio Grande do Sul podem ser considerados integrados no período considerado, e a terceira hipótese testa se esses mercados são perfeitamente integrados.

$\beta_{\mathrm{PR}}=0, \quad \beta_{\mathrm{SC}}=0, \quad \beta_{\mathrm{PR}}=\beta_{\mathrm{SC}}$

sendo que as duas primeiras buscam verificar se os mercados de milho no Paraná e em Santa Catarina podem ser considerados integrados no período enfocado, e a terceira hipótese testa se esses mercados são completamente integrados.

$\beta_{\mathrm{RS}}=0, \quad \beta_{\mathrm{SC}}=0, \quad \beta_{\mathrm{RS}}=\beta_{\mathrm{SC}}$

sendo que as duas primeiras visam testar se os mercados de milho no Rio Grande do Sul e em Santa Catarina podem ser considerados integrados no período analisado, e a terceira hipótese testa se esses mercados são totalmente integrados. 


\subsection{Fonte dos dados}

Os dados empregados neste estudo referem-se às séries mensais de preços do milho em grão, tipo 2, milho amarelo semiduro, para o período de outubro de 2002 a março de 2009. Esses dados foram obtidos do Centro de Estudos Avançados em Economia Aplicada (CEPEA), da ESALQ / USP. Tendo em vista que a região Sul é a maior produtora de milho no mercado nacional, ela foi escolhida neste estudo. A série de preços do Rio Grande do Sul foi obtida pela média mensal dos preços do milho nos municípios de Passo Fundo e Ijuí. Para representar o preço do milho em Santa Catarina, tomou-se a série de preços de Chapecó como representativa do Estado, enquanto a série de preços do milho no estado do Paraná foi determinada pela média mensal dos preços do milho em três praças de comercialização do estado: Ponta Grossa, norte do Paraná e Cascavel, que foram escolhidas devido à disponibilidade dos dados. Tais séries de preços foram convertidas em dólares e transformadas em logaritmos, de modo que os coeficientes obtidos se referem às elasticidades de transmissão de preços. Ademais, também é relevante destacar que esses preços não foram deflacionados, pois, conforme Siqueira (2007) e Cunha (2008), o deflacionamento incorpora uma tendência nos dados, logo não faz sentido incluir uma tendência antes da realização do teste de cointegração.

\section{Resultados e discussões}

A ilustração gráfica demonstra que as séries estudadas não apresentaram tendência uniforme ao longo do tempo, o que indica que tais séries de preços não são estacionárias, porém elas se movimentaram juntas no período considerado, indício que essas séries sejam cointegradas. Para confirmar essas evidências, foram realizados os testes econométricos de raiz unitária, estacionariedade e cointegração.

Quanto ao teste de raiz unitária, conforme mencionado, procedeu-se o teste HEGY de raiz unitária sazonal, visto que ele é mais robusto do que os testes convencionais de raiz unitária. Entretanto, antes disso, verificou se as séries continham sazonalidade determinística, cujos resultados se encontram na Tabela 1A do Apêndice. Tais resultados indicaram que esse componente não está presente nas séries analisadas no período considerado, visto que as constantes sazonais não foram estatisticamente significativas e a estatística F também se mostrou não significativa. 
FIGURA 1 - COMPORTAMENTO DOS PREÇOS DO MILHO NOS ESTADOS DO PARANÁ (LPPR), DE SANTA CATARINA (LPSC) E DO RIO GRANDE DO SUL (LPRS), OUTUBRO DE 2002 A MARÇO DE 2009.

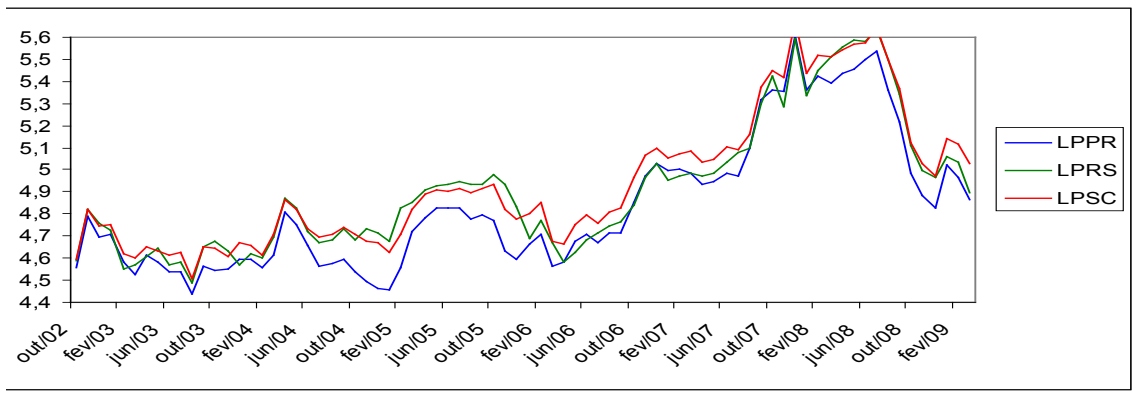

Os resultados do teste HEGY, contidos na Tabela 1, revelam que as séries de preços consideradas não apresentaram raiz unitária sazonal, conforme verificada pela significância estatística de $\pi$. Ademais, não se identificou raiz unitária semianual nem raízes unitárias complexas em todos os pares de $\pi$ i’s.

TABELA 1 - RESULTADOS DO TESTE DE RAIZ UNITÁRIA SAZONAL PARA AS SÉRIES MENSAIS DE PREÇOS DO MILHO NOS ESTADOS DO PARANÁ (LPPR), DO RIO GRANDE DO SUL (LPRS) E DE SANTA CATARINA (LPSC), OUTUBRO DE 2001 A MARÇO DE 2009

\begin{tabular}{|c|c|c|c|}
\hline Frequência/Coeficientes & & Séries & \\
\hline Trequericla/coentientes & LPPR & LPRS & LPSC \\
\hline $\begin{array}{l}\text { Lags } \\
0 / \pi^{(a)} \\
\pi / \pi_{2}^{1}\end{array}$ & $\begin{array}{c}0 \\
-0,45_{* * *}^{(b)} \\
-2,63^{*}\end{array}$ & $\begin{array}{c}\mathrm{O} \\
-0,4 \mathrm{O}^{(\mathrm{b})} \\
-2,28^{* *}\end{array}$ & $\begin{array}{c}\mathrm{O} \\
-0,3^{(\mathrm{b})} \\
-2,62^{* * *}\end{array}$ \\
\hline & $5,73^{* * *}$ & $7,37^{* * *}$ & $5,29^{* * *}$ \\
\hline$\frac{2 \pi}{3} / \pi_{3}$ e $\pi_{4}$ e $\pi_{6}$ & $6,60 * * *$ & $4,09 * *$ & $6,33^{* * *}$ \\
\hline$\frac{\pi}{3} / \pi_{7}$ e $\pi_{8}$ & $4,62^{* *}$ & $6,01^{* * *}$ & $4,98 * * *$ \\
\hline$\frac{5 \pi}{6} / \pi_{9}$ e $\pi_{10}$ & $6,14^{* * *}$ & $5,99^{* * *}$ & $5,66 * * *$ \\
\hline$\frac{\pi}{6} / \pi_{11}$ e $\pi_{12}$ & $3,02^{*}$ & $2,97^{*}$ & $2,79^{*}$ \\
\hline$\pi_{1}, \ldots, \pi_{12}$ & $44,49^{* * *}$ & $45,46^{* * *}$ & $41,94^{* * *}$ \\
\hline$\pi_{2}, \ldots, \pi_{12}$ & $46,93^{* * *}$ & $48,56^{* * *}$ & $44,49^{* * *}$ \\
\hline
\end{tabular}

FONTE: Dados da pesquisa.

Nota: ${ }^{(a)} \mathrm{O}$ número de defasagens foi baseado no critério de Schwarz; ${ }^{(b)}$ Indica não rejeição da hipótese nula; $\left({ }^{* *}\right),\left(^{* *}\right)$ e $\left(^{*}\right)$ indicam rejeição de Ho a $1 \%, 5 \%$ e $10 \%$, respectivamente, cujos valores críticos foram obtidos por Franses \& Hobijn (1990).

A partir da inspeção visual das séries, observa-se uma nítida mudança estrutural a partir de 2006. Esse comportamento ascendente dos preços domésticos 
do milho pode ser resultante da elevação de seus preços no mercado internacional. Conforme Perez et al. (2007), os preços desse grão aumentaram 89,6\% no mercado externo, em 2006. Portanto, com base nessas evidências, realizou-se o teste de Chow para verificar se de fato houve quebra estrutural em 2006. Considerando um modelo com intercepto e tendência, os resultados contidos na Tabela 2 mostram que os valores estimados foram menores, em módulo, que seus valores críticos nas três séries analisadas, logo não se rejeita a hipótese nula de ausência de mudança estrutural.

TABELA 2 - RESULTADOS DO TESTE DE QUEBRA ESTRUTURAL PARA AS SÉRIES MENSAIS DE PREÇOS DO MILHO NOS ESTADOS DO PARANÁ (LPPR), DO RIO GRANDE DO SUL (LPRS) E DE SANTA CATARINA (LPSC), OUTUBRO DE 2001 A MARÇO DE 2009

\begin{tabular}{ccccc}
\hline Séries & Lags $^{(a)}$ & Valor esti- $^{\prime}$ & \multicolumn{2}{c}{ Valor crítico $^{(b)}$} \\
& & mado & $1 \%$ & $5 \%$ \\
\hline LPPR & 0 & $-1,71$ & $-3,55$ & $-3,03$ \\
LPRS & 0 & $-1,64$ & $-3,55$ & $-3,03$ \\
LPSC & 0 & $-1,82$ & $-3,55$ & $-3,03$ \\
\hline
\end{tabular}

FONTE: Dados da pesquisa.

Nota: ${ }^{(a)} \mathrm{O}$ número de defasagens foi baseado no critério de Schwarz; ${ }^{\left({ }^{b}\right)}$ Esses valores críticos foram obtidos por Lanne et al. (2002).

Como as séries de preços do milho, nos três estados analisados, são integradas na mesma ordem, nesse período considerado buscou-se verificar se elas tinham relacionamento de longo prazo. No entanto, antes de proceder aos testes de cointegração, deve-se identificar o número de defasagens que deve ser incluído no modelo VAR. Neste estudo, considerou-se uma defasagem para cada par de séries de preços, conforme sugerido pelos critérios de Schwarz e Hannan-Quinn.

O método VAR possibilita determinar o poder de explicação de cada variável considerada sobre as demais por meio da decomposição da variância dos erros de previsão, conforme apresentado na Tabela 3. Com relação à variável LPPR, verifica-se que após doze meses da incidência de um choque não antecipado sobre essa variável, a maior parte (97,31\%) de sua decomposição da variância dos erros de previsão pode ser atribuída a ela própria, sendo um pequeno percentual decorrente das outras duas variáveis. Ademais, a variável LPPR também exerceu grande poder de explicação na decomposição da variância dos erros de previsão das variáveis LPRS e LPSC. Esse resultado é um indício da importância do Paraná como mercado central, que transmite alterações de preços aos demais estados da região Sul. 
TABELA 3 - DECOMPOSIÇÃO DA VARIÂNCIA DOS ERROS DE PREVISÃO, EM PERCENTAGEM, DE LPPR, LPRS E LPSC PARA AS VARIÁVEIS LPPR, LPRS E LPSC, OUTUBRO DE 2002 A MARÇO DE 2009

\begin{tabular}{|c|c|c|c|c|c|c|c|c|c|}
\hline \multirow[t]{2}{*}{$\begin{array}{l}\text { Pe- } \\
\text { río- } \\
\text { do }\end{array}$} & \multicolumn{3}{|c|}{$\begin{array}{l}\text { Decomposição da variân- } \\
\text { cia dos erros de previsão } \\
\text { de LPPR }\end{array}$} & \multicolumn{3}{|c|}{$\begin{array}{l}\text { Decomposição da } \\
\text { variância dos erros de } \\
\text { previsão de LPRS }\end{array}$} & \multicolumn{3}{|c|}{$\begin{array}{l}\text { Decomposição da } \\
\text { variância dos erros de } \\
\text { previsão de LPSC }\end{array}$} \\
\hline & LPPR & LPRS & $\overline{\text { LPSC }}$ & LPPR & LPRS & LPSC & LPPR & LPRS & LPSC \\
\hline 1 & 10 & 0,00 & 0,00 & 67,98 & 32,02 & 0,00 & 93,87 & 2,17 & 3,96 \\
\hline 3 & 96,33 & 2,33 & 1,34 & 86,65 & 13,23 & 0,12 & 97,05 & 1,65 & 1,30 \\
\hline 6 & 96,92 & 2,21 & 0,87 & 91,80 & 8,04 & 0,16 & 97,96 & 1,11 & 0,93 \\
\hline 9 & 97,21 & 1,94 & 0,85 & 93,36 & 6,20 & 0,44 & 98,04 & 0,88 & 1,08 \\
\hline 12 & 97.31 & 1,72 & 0,97 & 94,01 & 5,27 & 0,72 & 97,98 & 0,74 & 1,28 \\
\hline
\end{tabular}

Fonte: Dados da pesquisa.

As elasticidades da função de impulso-resposta também podem ser obtidas a partir do método VAR. Elas revelam as respostas das variáveis LPPR, LPRS e LPSC à própria variável e às demais. Os dados da Tabela 4 apontam que um desvio-padrão em LPPR hoje causará, respectivamente, 0,052; -0,002 e o,008 desvios-padrões sobre ela própria e sobre LPRS e LPSC, no décimo segundo mês. Por sua vez, um desvio-padrão em LPRS hoje repercutirá em 0,001; 0,052 e 0,008 desvios-padrões sobre ela própria e sobre LPPR e LPSC, respectivamente, no décimo segundo mês. Raciocínio análogo pode ser feito para os impactos de choques de LPSC sobre si próprio e sobre as variáveis LPPR e LPRS. Esses resultados demonstram que um choque não antecipado sobre os preços do milho, nos estados sulinos, geraria pequenos efeitos no curto prazo, ou seja, isso significaria que os desequilíbrios transitórios seriam corrigidos lentamente.

TABELA 4 - ELASTICIDADES DA FUNÇÃO DE IMPULSO-RESPOSTA DE LPPR, LPRS E LPSC, OUTUBRO DE 2002 A MARÇO DE 2009

\begin{tabular}{|c|c|c|c|c|c|c|c|c|c|}
\hline \multirow{2}{*}{$\begin{array}{l}\text { Pe- } \\
\text { río- } \\
\text { do }\end{array}$} & \multicolumn{3}{|c|}{$\begin{array}{l}\text { Efeitos de choques de } \\
\text { LPPR }\end{array}$} & \multicolumn{3}{|c|}{$\begin{array}{l}\text { Efeitos de choques de } \\
\text { LPRS }\end{array}$} & \multicolumn{3}{|c|}{$\begin{array}{l}\text { Efeitos de choques de } \\
\text { LPSC }\end{array}$} \\
\hline & LPPR & LPRS & LPSC & LPPR & LPRS & LPSC & LPPR & LPRS & LPSC \\
\hline 1 & 0,092 & 0,000 & 0,000 & 0,075 & 0,051 & 0,000 & 0,083 & 0,013 & 0,017 \\
\hline 3 & 0,091 & $-0,014$ & $-0,012$ & 0,081 & 0,011 & $-0,005$ & 0,084 & $-0,006$ & $-0,001$ \\
\hline 6 & 0,072 & $-0,009$ & 0,00 & 0,068 & 0,003 & 0,0 & 0,071 & $-0,004$ & 0,007 \\
\hline 9 & 0,060 & $-0,005$ & & 0,060 & 0,0 & 0,0 & 0,061 & $-0,002$ & 0,009 \\
\hline 12 & 0,052 & $-0,002$ & 0,008 & 0,052 & 0,001 & 0,008 & 0,053 & $-0,001$ & 0,008 \\
\hline
\end{tabular}

FONTE: Dados da pesquisa.

Em seguida, realizou-se o teste de cointegração de Johansen, cujo modelo adotado considera que essas séries analisadas não tinham tendências determinísticas, já que as séries não apresentaram tendência definida como se observou na Figura 1. Como se está interessado em verificar o relacionamento de longo prazo entre esses mercados regionais, optou-se, neste trabalho, pela especificação do modelo sem tendência determinística e sem intercepto, pois, 
ao empregar a especificação com intercepto, as séries de preço do milho não apresentaram relação de cointegração.

A Tabela 5 contém os resultados do teste de cointegração. Esses resultados indicam que as séries de preços do milho entre os estados do Paraná e do Rio Grande do Sul e entre os estados do Rio Grande do Sul e de Santa Catarina apresentam um vetor de cointegração, já que se rejeita a hipótese nula, a 5\%, de que não há nenhum vetor de cointegração, conforme mostram os testes do traço e do máximo autovalor. Já com relação às séries de preços do milho entre os estados do Paraná e de Santa Catarina, os resultados dos testes apontam para a aceitação da hipótese nula, sinalizando que não há vetor de cointegração.

TABELA 5 - RESULTADOS DO TESTE DE COINTEGRAÇÃO DE JOHANSEN PARAAS SÉRIES MENSAIS DE PREÇOS DO MILHO NOS ESTADOS DO PARANÁ (LPPR), RIO GRANDE DO SUL (LPRS) E SANTA CATARINA (LPSC), OUTUBRO DE 2002 A MARÇO DE 2009

\begin{tabular}{cccccc}
\hline $\begin{array}{c}\text { Séries relacio- } \\
\text { nadas }\end{array}$ & $\begin{array}{c}\text { Hipóte- } \\
\text { se nula }\end{array}$ & $\begin{array}{c}\text { Teste do } \\
\text { Traço }\end{array}$ & $\begin{array}{c}\text { Valor } \\
\text { Crítico } \\
(5 \%)\end{array}$ & $\begin{array}{c}\text { Teste do } \\
\text { máximo } \\
\text { autovalor }\end{array}$ & $\begin{array}{c}\text { Valor } \\
\text { Crítico } \\
(5 \%)\end{array}$ \\
\hline LPPR x LPRS & $\mathrm{r}=0$ & $13,1985^{*}$ & 12,3209 & $13,1392^{*}$ & 11,2248 \\
& $\mathrm{r} \leq 1$ & 0,0592 & 4,1299 & 0,0592 & 4,1299 \\
\hline LPPR x LPSC & $\mathrm{r}=0$ & 11,0162 & 12,3209 & 11,0052 & 11,2248 \\
& $\mathrm{r} \leq 1$ & 0,0110 & 4,1299 & 0,0110 & 4,1299 \\
\hline LPRS x LPSC & $\mathrm{r}=0$ & $14,2457^{*}$ & 12,3209 & $14,0126^{*}$ & 11,2248 \\
& $\mathrm{r} \leq 1$ & 0,2331 & 4,1299 & 0,2331 & 4,1299 \\
\hline
\end{tabular}

FONTE: Dados da pesquisa.

* indica rejeição da hipótese nula a 5\% de significância.

As equações de cointegração das séries LPPR x LPRS e LPRS x LPSC são dadas por: $\mathrm{LPPR}=0,9844 \mathrm{LPRS}$ e LPRS $=0,9940$ LPSC. A primeira equação sinaliza que, ceteris paribus, cada variação de $1 \%$ no preço do milho no Rio Grande do Sul gerou uma variação de 0,98\% no preço do milho no Paraná, e a segunda equação indica que $0,99 \%$ das variações de preços do milho, no longo prazo, ocorridas em Santa Catarina, foram repassadas para o preço do milho no Rio Grande do Sul, no período de outubro de 2002 a março de 2009.

A partir desses resultados da elasticidade de transmissão do preço do milho, observa-se a predominância da Lei do Preço Único nesses mercados regionais, tendo em vista que seu coeficiente foi bem próximo da unidade. No entanto, segundo Costa e Ferreira Filho (2000) e Chiodi (2006), devem ser impostas restrições aos parâmetros $\beta$, para testar a perfeita integração do mercado, ou seja, para confirmar se essa lei se manterá.

A Tabela 6 contém os resultados dos testes de hipóteses sobre os parâmetros $\beta$ para as séries que associam os preços do milho nos estados do Paraná e do Rio Grande do Sul, assim como para as séries que relacionam os preços do 
milho nos estados do Rio Grande do Sul e de Santa Catarina. Os dados mostram que a hipótese nula acerca dos parâmetros $\beta_{\mathrm{PR}}$ e $\beta_{\mathrm{RS}}$ deve ser rejeitada, pois seus valores da razão de verossimilhança excederam seus respectivos valores críticos de $5 \%$, logo, essas variáveis participaram das relações de longo prazo, o que indica que variações de preços de milho ocorridas em um mercado foram repassadas para outro no longo prazo. Essa evidência também é observada nos mercados do Rio Grande do Sul e de Santa Catarina, o que revela que essas séries de preços foram significativamente importantes no estabelecimento do padrão de equilíbrio de longo prazo.

TABELA 6 - TESTE DE SIGNIFICÂNCIA DE RESTRIÇÃO AO PARÂMETRO DE LONGO PRAZO ( $\beta$ ) DO VETOR DE COINTEGRAÇÃO DAS SÉRIES MENSAIS DE PREÇOS DO MILHO NOS ESTADOS DO PARANÁ (LPPR), DO RIO GRANDE DO SUL (LPRS) E DE SANTA CATARINA (LPSC), OUTUBRO DE 2002 A MARÇO DE 2009

\begin{tabular}{cccc}
\hline Séries relacionadas & Hipótese nula & $\begin{array}{c}\text { Razão de Verossi- } \\
\text { milhança }\end{array}$ & $\begin{array}{c}\text { Valor } \\
\text { crítico } \\
(5 \%)\end{array}$ \\
\hline LPPR x LPRS & $\beta_{\mathrm{PR}}=0$ & $13,07^{*}$ & 3,84 \\
& $\beta_{\mathrm{RS}}=0$ & $13,05^{*}$ & 3,84 \\
LPRS x LPSC & $\beta_{\mathrm{PR}}=\beta_{\mathrm{RS}}$ & $13,06^{*}$ & 3,84 \\
& $\beta_{\mathrm{RS}}=\mathrm{O}$ & $13,75^{*}$ & 3,84 \\
& $\beta_{\mathrm{SC}}=\mathrm{O}$ & $13,74^{*}$ & 3,84 \\
& $\beta_{\mathrm{RS}}=\beta_{\mathrm{SC}}$ & $13,75^{*}$ & 3,84 \\
\hline
\end{tabular}

FONTE: Dados da pesquisa.

* indica rejeição a 5\% de significância.

Como se verificou que esses mercados são integrados, então deve-se testar o grau de integração, ou seja, testar se essas séries de preços são perfeitamente integradas. A partir das hipóteses nulas $\beta_{\mathrm{PR}}=\beta_{\mathrm{RS}}$ e $\beta_{\mathrm{RS}}=\beta_{\mathrm{SC}}$, constata-se que ambos os pares de mercados não foram perfeitamente integrados no período considerado, já que os valores da razão de verossimilhança foram maiores do que seus respectivos valores críticos de $5 \%$. Portanto, a Lei do Preço Único não foi perfeitamente verificada no mercado de milho nesses estados analisados. Esses resultados são corroborados com os encontrados por Chiodi (2006). Deve-se enfatizar que este é um resultado comum na literatura, uma vez que a perfeita integração requer um funcionamento do mercado em condições muito próximas às de concorrência perfeita.

Tendo em vista que as séries de preços entre os estados do Paraná e do Rio Grande do Sul, assim como as séries de preços do Rio Grande do Sul e de Santa Catarina, apresentaram um vetor de cointegração, elas convergiram para uma condição de equilíbrio de longo prazo, tornando-se relevante a estimação do Modelo de Correção de Erro (VEC), cujos valores estimados se encontram na Tabela 7. 
TABELA 7 - ESTIMAÇÃO DO VEC PARA AS SÉRIES MENSAIS DE PREÇOS DO MILHO NOS ESTADOS DO PARANÁ (LPPR), DO RIO GRANDE DO SUL (LPRS) E DE SANTA CATARINA (LPSC), OUTUBRO DE 2002 A MARÇO DE 2009

\begin{tabular}{ccccc}
\hline $\begin{array}{c}\text { Séries relacio- } \\
\text { nadas }\end{array}$ & $\begin{array}{c}\text { Variável } \\
\text { explicativa }\end{array}$ & $\begin{array}{c}\text { Coeficiente } \\
\text { estimado }\end{array}$ & Estatística t & $\begin{array}{c}\text { Desvio } \\
\text { padrão }\end{array}$ \\
\hline LPPR x LPRS & $\mathrm{u}_{\mathrm{t}-1}$ & 0,0679 & 0,4789 & 0,1417 \\
LPRS x LPSC & $\mathrm{u}_{\mathrm{t}-1}$ & $-0,4546$ & $-2,2403$ & 0,2029 \\
\hline
\end{tabular}

FONTE: Dados da pesquisa.

Na Tabela 7, verifica-se que, na série LPPR x LPRS, 6,79\% do desequilíbrio de curto prazo referente à trajetória de longo prazo foi corrigido a cada mês, o que indica que necessitaria, em média, de catorze meses para corrigi-lo, isto é, esses desequilíbrios foram corrigidos muito lentamente. No entanto, os desequilíbrios de curto prazo foram corrigidos rapidamente na série LPRS x LPSC, já que, em média, dois meses seriam suficientes para removê-los.

\section{Conclusões}

Os resultados demonstraram a presença de relações de equilíbrio de longo prazo entre os preços do milho praticados entre os estados do Paraná e do Rio Grande do Sul e entre os estados do Rio Grande do Sul e de Santa Catarina, porém não se verificou relação de cointegração entre os estados do Paraná e de Santa Catarina.

As elasticidades da função de impulso-resposta mostraram que há longa defasagem temporal até que o equilíbrio de longo prazo seja restabelecido, ou seja, os desequilíbrios transitórios foram corrigidos lentamente em todos os mercados analisados. Com relação à decomposição da variância dos erros de previsão, observou-se que a variável relativa aos preços do Paraná (LPPR) foi responsável pela maior parcela de explicação na decomposição da variância dos erros de previsão não apenas em sua própria variável, como também nas séries de preços nos estados do Rio Grande do Sul (LPRS) e de Santa Catarina (LPSC).

Este estudo também mensurou as elasticidades de transmissão de preços do milho entre o Paraná e o Rio Grande do Sul e entre o Rio Grande do Sul e Santa Catarina, o que indica que variações em um mercado, no longo prazo, foram transmitidas para o outro mercado considerado, durante o período de outubro de 2002 a março de 2009. Esses resultados revelam que a Lei do Preço Único prevaleceu nesses mercados, porém quando foram impostas restrições ao parâmetro de longo prazo $\beta$, tais mercados não foram considerados 
perfeitamente integrados, já que a hipótese de perfeita integração entre eles foi rejeitada. Portanto, a Lei do Preço Único não foi completamente verificada no mercado de milho no Sul brasileiro, no período considerado.

Ademais, verificou-se, por meio da estimação do modelo de correção de erro, que os desequilíbrios de curto prazo foram corrigidos de forma muito mais rápida entre as séries de preços do Rio Grande do Sul e de Santa Catarina, do que as séries de preços que relacionam Paraná e Rio Grande do Sul.

Por fim, sugerem-se, em estudos posteriores, que sejam levados em consideração outros fatores que influenciam os preços nos mercados regionais de milho, como, por exemplo, o impacto de variações das cotações da taxa de câmbio nesse mercado.

\section{Referências}

ALVES, L. R. \& CARDOSO, C. E. L. \& FELIPE, F. I. \& CAMPION, M. T. (2006). "Causalidade e transmissão entre os preços de mandioca, trigo, milho e seus derivados no Paraná”. Revista de Economia e Agronegócio 4 (3): 313-342.

ALVES, L. R. \& RIZATO, M. \& ZINSLY, A. A. \& MAGGIAN, R. \& RESENDE, K. \& CAMARGO, A. L. (2009). "Informações de Mercado". Agromensal Abril 2009 - ESALQ/BM\&FBovespa. URL :http://www.cepea.esalq.usp.br/agromensal/2009/04_abril/milho.htm. Acesso em: 22/09/2009.

ARDENI, P. G. (1989). "Does the Law of One Price really hold for commodity prices?” American Journal of Agricultural Economics 71 (3): 661-669.

BACCHI, M. R. P. (1994). Previsão de preços de bovino, suíno e frango com modelos de séries temporais. Piracicaba: Tese de Doutorado, Universidade de São Paulo, Programa de Pós-Graduação em Economia Aplicada.

BITTENCOURT, M. V. L. \& BARROS, G. S. C. (1996). "Relações de preço de frango nas Regiões Sul e Sudeste do Brasil”. Revista de Economia e Sociologia Rural 34 (3 е 4): 147-169.

BUONGIORNO, J. \& UUSIVUORI, J. (1992). “The Law of One Price in the trade of forest products: Cointegration Tests for U.S. exports of pulp and paper”. Forest Science 38 (3): 539-553.

CHIODI, L. (2006). Integração espacial no mercado brasileiro de milho. Piracicaba: Dissertação de Mestrado, Universidade de São Paulo, Programa de PósGraduação em Economia Aplicada.

COELHO, A. B. (2004). “A cultura do algodão e a questão da integração entre preços internos e externos”. Revista de Economia e Sociologia Rural 42 (1): 153-169.

CONCEIÇÃO, J. C. P. R. (2002). Contribuição dos novos instrumentos de comercialização (Contratos de Opção e PEP) para estabilização de preço e renda agrícolas. Brasília: IPEA (Texto para Discussão, 927).

COSTA, S. M. A. L. \& FERREIRA FILHO, J. B. S. (2000). "Liberalização comercial no Brasil e integração nos mercados de commodities agrícolas: os mercados de algodão, milho e arroz". Revista de Economia e Sociologia Rural 38 (2): 41-70. 
CUNHA, D. A. (2008). Integração de preços no mercado internacional de café. Viçosa: Dissertação de Mestrado, Universidade Federal de Viçosa, Programa de Pós-Graduação em Economia Aplicada.

DUARTE, J. O. (2007). “Cultivo do milho - Mercado e comercialização”. Embrapa Milho e Sorgo. Sistemas de Produção. URL: http://www.cnpms.embrapa.br/ publicacoes/milho/mercado.htm. Acesso em: 19/04/2009.

ENDERS, W. (1995). Applied Econometric Time Series. Nova York: John Wiley \& Sons.

ENGLE, R. F. \& GRANGER, C. W. (1987). "Co-integration and error-correction: representation, estimation and testing”. Econometrica 55 (2): 251-276.

FACKLER, P. L. \& GOODWIN, B. K. (2001). "Spatial price analysis”. In: GARDNER, B. L. \& RAUSSER, G. C. (eds.). Handbook of Agricultural Economics. Amsterdam: Elsevier, pp. 971-1024.

FRANSES, P. H. \& HOBIJN, B. (1997). "Critical values for unit root tests in seasonal time series”. Journal of Applied Statistics 24 (1): 25-46.

GONZALO, J. (1994). "Five alternative methods of estimating long-run equilibrium relationships". Journal of Econometrics 60: 203-233.

HARRIS, R. I. D. (1995). Using cointegration analysis in econometric modelling. London: Prentice Hall.

HYLLEBERG, S. \& ENGLE, R. F. \& GRANGER, C. W. J. \& YOO, B. S. (1990). "Seasonal integration and cointegration”. Journal of Econometrics 44 (1-2): 215-238.

INSTITUTO BRASILEIRO DE GEOGRAFIA E ESTATÍSTICA - IBGE (2007). Produção Agrícola Municipal. Rio de Janeiro.

JUNG, C. \& DOROODIAN, K. (1994). "The Law of One Price for U.S. softwood lumber: a Multivariate Cointegration Test”. Forest Science 40 (4):595-600.

KRUGMAN, P. R. \& OBSTFELD, M. (2005). Economia Internacional: teoria e política. São Paulo: Pearson Addison Wesley.

LANNE, M. \& LÜTKEPOHL, H. \& SAIKKONEN, P. (2002). "Comparison of unit root tests for time with level shifts”. Journal of Time Series Analysis 23: 667-685.

LIMA, S. M. A. \& BURNQUIST, H.L. (1997). "Lei do preço único no mercado internacional: testes empíricos para exportações do complexo soja (grãos e farelo)". In: XXXV Congresso Brasileiro de Economia e Sociologia Rural, Anais. Natal, CD-ROM.

MARGARIDO, M. A. \& BUENO, C. R. F. \& MARTINS, V. A. \& CARNEVALLI, L. B. (2004). "Análise dos efeitos preço e câmbio sobre o preço do óleo de soja na cidade de São Paulo: uma aplicação do modelo VAR”. Pesquisa \& debate 15 (1): 69-106.

MAYORGA, R. O. (2006). Análise de transmissão de preços do mercado atacadista de melão do Brasil. Fortaleza: Dissertação de Mestrado, Universidade Federal do Ceará, Programa de Pós-Graduação em Economia Rural.

MUNDLAK; Y. \& LARSON, D. F. (1992). “On the transmission of world agricultural prices”. The World Bank Economic Review 6(3):399-422.

NOGUEIRA, F. T. P. \& AGUIAR, D. R. D. \& LIMA, J. E. (2005). “Integração espacial 
no mercado brasileiro de café arábica”. Nova Economia 15 (2): 91-112.

PEREZ, L. H. \& SACHS, R. C. C. \& RESENDE, J. V. (2007). "Milho: demanda por etanol eleva cotações”. Análises e Indicadores do Agronegócio 2 (5). URL: http:// www.iea.sp.gov.br/out/verTexto.phd?codTexto=8945. Acesso em: 23/o9/2009.

PORTAL DO AGRONEGÓCIO (2009). "Paraná pede intervenção da União para amparar venda de milho e trigo". URL: http://www.portaldoagronegocio.com. br/conteudo.php?=31491. Acesso em: 22/09/2009.

ROSADO, P. L. (2006). Integração espacial entre os mercados brasileiros de suínos. Viçosa: Tese de Doutorado, Universidade Federal de Viçosa, Programa de Pós-Graduação em Economia Aplicada.

SAMPAIO, L.M.B. \& LIMA, R.C. \& SAMPAIO, Y. (2005). "Inter-relação das exportações de Brasil, Argentina e EUA: uma abordagem utilizando autoregressão vetorial”. In: XLIII Congresso da Sociedade Brasileira de Economia e Sociologia Rural, Anais. Ribeirão Preto, CD-ROM.

SANTOS, V. F. \& PEREIRA, M. W. G. \& BRAGA, M. J. \& VIEIRA, W. C. (2007). "Análise do preço do milho nos mercados externo e interno". Revista de Política Agrícola 16 (3): 76-84.

SILVEIRA, A. M. A. (2004). Relação entre os preços de açúcar nos mercados doméstico e internacional. Piracicaba: Dissertação de Mestrado, Universidade de São Paulo, Programa de Pós-Graduação em Economia Aplicada.

SIQUEIRA, K. B. (2007). The dynamics of farm milk price formation in Brazil. Viçosa: Tese de Doutorado, Universidade Federal de Viçosa, Programa de PósGraduação em Economia Aplicada.

VERBEEK, M. (2000). A guide to modern econometrics. Chichester: John Wiley \& Sons.

Recebido em: 14 de maio de 2009

Primeira resposta em: 21 de setembro de 2009

Aceite em: 12 de novembro de 2009 


\section{APÊNDICE}

TABELA 1A - TESTE DE SAZONALIDADE DETERMINÍSTICA PARA AS SÉRIES MENSAIS DE PREÇOS DO MILHO NOS ESTADOS DO PARANÁ (LPPR), DO RIO GRANDE DO SUL (LPRS) E DE SANTA CATARINA (LPSC), OUTUBRO DE 2001 A MARÇO DE 2009

\begin{tabular}{crrr}
\hline Meses & \multicolumn{3}{c}{ Constantes sazonais } \\
\cline { 2 - 4 } & LPPR & LPRS & \multicolumn{1}{c}{ LPSC } \\
\hline Janeiro & $-0,0086$ & $-0,0459$ & $-0,0137$ \\
Fevereiro & $-0,0102$ & $-0,0242$ & $-0,0097$ \\
Março & $-0,0290$ & $-0,0283$ & $-0,0207$ \\
Abril & 0,0208 & 0,0051 & 0,0023 \\
Maio & 0,0344 & 0,0207 & 0,0165 \\
Junho & 0,0307 & 0,0093 & 0,0150 \\
Julho & 0,0139 & 0,0274 & 0,0158 \\
Agosto & $-0,0104$ & $-0,0040$ & $-0,0104$ \\
Setembro & 0,0283 & 0,0436 & 0,0414 \\
Outubro & $-0,0368$ & $-0,0120$ & $-0,0226$ \\
Novembro & $-0,0260$ & $-0,0032$ & $-0,0185$ \\
Dezembro & $-0,0071$ & 0,0117 & 0,0047 \\
\hline Estatística F & $0,0367^{*}$ & $0,0404^{*}$ & $0,0224^{*}$ \\
\hline
\end{tabular}

FONTE: Dados da pesquisa.

* indica não rejeição de Ho. 